\title{
Dissolution Rates at Dislocation Sites on the (111) Surface of Cu Single Crystals Etched in Young's Solution at 290 K*
}

\author{
By Shigeo Sugawara** and Jirô Watanabé**
}

\begin{abstract}
The net dissolution rate at dislocation sites on the $\mathrm{Cu}$ (111) surface etched in Young's etchant $\left(1 \mathrm{kmol} \cdot \mathrm{m}^{-3}\left(\mathrm{NH}_{4}\right)_{2} \mathrm{~S}_{2} \mathrm{O}_{8}, 6 \mathrm{kmol} \cdot \mathrm{m}^{-3} \mathrm{NH}_{4} \mathrm{OH}, 0.3 \mathrm{kmol} \cdot \mathrm{m}^{-3} \mathrm{NH}_{4} \mathrm{Br}\right)$ has been determined from measurements of the dissolved thickness of the matrix surface as well as the three-dimensional size of dislocation etch pits. The etch pit size was measured by a replica electron microscopy. After etching time of $5 \mathrm{~s}$, the width and depth of the etch pits increased at constant rates of $\dot{h}$ and $\dot{d}$, respectively, leaving the side slope unchanged. The dissolved thickness of the matrix surface was determined by an interferometric measurement of the step height which was produced along the boundary between the masked surface and the exposed surface. It was found that the matrix surface dissolved also at a constant rate $s$ after $5 \mathrm{~s}$ etching. The net dissolution rate normal to the surface at a dislocation site, $v_{d}$, and that parallel to the surface, $v_{h}$, were estimated to be 1.7 times as large as the measured growth rates of etch pits $\dot{d}$ and $\dot{h}$, respectively. This fact indicates that dissolution of the matrix surface must not be neglected in describing the dissolution at dislocation sites. Using the values of $v_{d}, v_{h}$ and $\dot{s}$, the nucleation rates of two-dimensional etch pit nuclei at dislocation sites and matrix surface were obtained as $2.9 \times 10^{2} \mathrm{~s}^{-1}$ per dislocation and $8.7 \times 10^{6} \mu \mathrm{m}^{-2} \cdot \mathrm{s}^{-1}$, respectively.
\end{abstract}

(Received June 2, 1984)

Keyzords: copper single crystal, crystal dissolution, dislocation etch pit, dissolution rate, replica, electron microscopy, interferometry

\section{Introduction}

When a crystal containing dislocations is etched with a suitable etchant, etch pits are formed at sites where dislocations intersect with a low-index crystal surface. The initiation of dislocation etch-pits has been considered as a nucleation process analogous to the crystal growth $^{(1)}$. A two-dimensional nucleus of etch pit with one atom height is preferentially formed at a dislocation site due to its excess strain energy, and subsequently it grows laterally by a movement of the resultant steps arising from a removal of atoms at its kink sites. Repeated nucleation and growth of a two-dimensional etch pit nucleus at a dislocation can lead to the formation of an etch pit. The formation of visible etch pits depends on the nucleation rate of etch pit nucleus at the dislocation and the rate of lateral motion of the steps. These two quantities are reflected in the linear dissolution

* This paper was originally published in Japanese in J. Japan Inst. Metals, 47 (1983), 820.

** Department of Metallurgy, Mining College, Akita University, Akita 010, Japan. rate normal to the crystal surface at the dislocation, $v_{d}$, and the lateral one parallel to the crystal surface, $v_{h}$. If $v_{d} \ll v_{h}$, very shallow pits would be formed which could not be observed under an optical microscope because it would lack contrasts when illuminated. It has been found that readily observable pits can be obtained when $v_{d} / v_{h} \gtrsim 0.1$. Therefore, a suitable etchant to reveal visible etch pits must contain an optimum amount of step poison to decrease $v_{h}$ in addition to an optimum amount of oxidizing agent to increase $v_{d}$. For the above mechanism, quantitative treatments have been developed on the basis of thermodynamical considerations $^{(2)-(4)}$ (thermodynamical theory or two-dimensional nucleation theory).

On the other hand, it has been usually considered that the kinetics for dislocation etch pitting involves the following processes; (1) transport of reactive chemical species in the etchant onto a crystal surface, (2) adsorption at the surface, (3) surface diffusion toward active sites, such as dislocation sites or kink positions in monatomic steps, (4) chemical reaction at the interface and (5) desorption and transport of chemical products from the sur- 
face. The slowest process among them would become the rate controlling one. The process (1) or (5) has been assumed to be predominant in the diffusion theory of crystal dissolution, and the process (2) in the adsorption theory ${ }^{(4)}$.

In order to verify the above kinetic theories for dissolution at dislocation sites, it is needless to say that a precise measurement of dissolution rates at dislocation sites, $v_{h}$ and $v_{d}$, is important. However, the most interest has so far been turned only to the measurement of the growth rates of width and/or depth of dislocation etch-pit ${ }^{(5)-(8)}$, and the simultaneous dissolution of matrix surfaces has been neglected. Therefore, the application of such treatment to the kinetic theories becomes extremely difficult. In the present paper we present the measurements of both the dissolved thickness of matrix surface and the width and depth of dislocation etch pits on the $\mathrm{Cu}$ (111) surface etched in Young's solution $(290 \mathrm{~K})$ for various periods and estimate the net dissolution rates $v_{h}$ and $v_{d}$ at the dislocation sites.

\section{Experimental Procedure}

The method of preparing a $\mathrm{Cu}(99.998 \%$ in purity) single crystal and revealing its (111) surface is similar to that reported in a previous paper $^{(9)}$; a mirrorlike surface which coincided with the (111) plane within $0.0087 \mathrm{rad}\left(0.5^{\circ}\right)$ was obtained by acid cutting and successive chemical and electrolytical polishing of a Czochralski $\mathrm{Cu}$ [111] crystal. Two pieces of vinyl tape were affixed on a small part of the (111) surface, as illustrated in Fig. 1. The specimen was dipped in a distilled water maintained

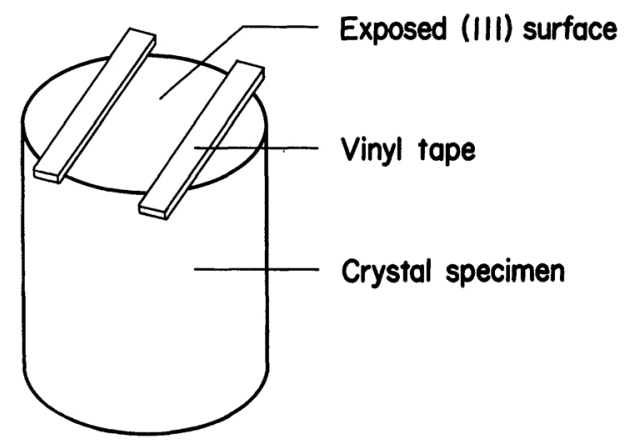

Fig. 1 Specimen surface masked by vinyl tapes. at a temperature of $290 \pm 0.1 \mathrm{~K}$ with the use of a thermostat ${ }^{(10)}$ for about $120 \mathrm{~s}$ (2 min) so that the temperature of specimen surface might coincide with that of etching solution. The etchant used was the Young's solution(11) (1 $\mathrm{kmol} \cdot \mathrm{m}^{-3}\left(\mathrm{NH}_{4}\right)_{2} \mathrm{~S}_{2} \mathrm{O}_{8}, 6 \mathrm{kmol} \cdot \mathrm{m}^{-3} \mathrm{NH}_{4}$ $\mathrm{OH}, 0.3 \mathrm{kmol} \cdot \mathrm{m}^{-3} \mathrm{NH}_{4} \mathrm{Br}$ ), which was prepared just before etching (600-1200 s (10$20 \mathrm{~min}$ ) before). The etching time was 5, 10, 15, 20 and $25 \mathrm{~s}$. After etching, the specimen was immediately washed with water, and vinyl tapes were stripped from the crystal surface. Then, the specimen was washed again and dried in a stream of nitrogen.

Since the part of surface masked with the vinyl tape remains undissolved during etching, a step can be produced along a boundary between the etched surface and the unexposed surface, as shown in an illustration of Fig. 2; the step height $s$ is equivalent to a dissolved amount of the matrix surface. Multi-beam interferometric observation (two-beam interferometric observation for supplement) was made to measure the step height $s$ with the use of Surface Finish Microscope (Nippon Kogaku K.K.). The measurement was carried out at 10 points of $0.5 \mathrm{~mm}$ intervals along the boundary line for every case of three experiments at

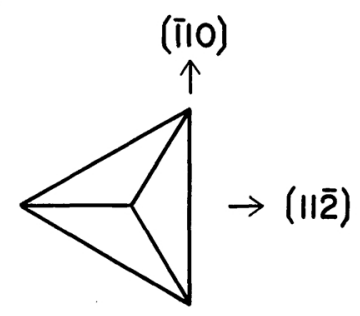

(a) Normal view

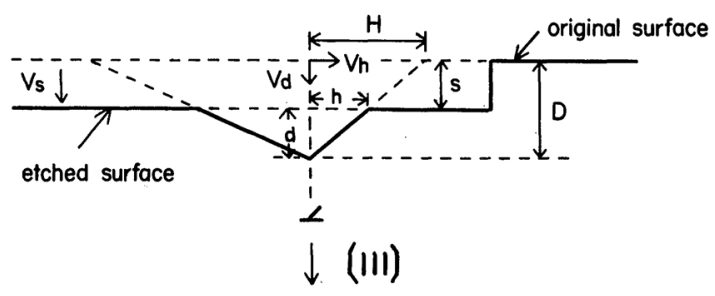

(b) Sectional view

Fig. 2 Schematic illustration of the relation between dissolution of a matrix surface and that of a dislocation etch pit. 


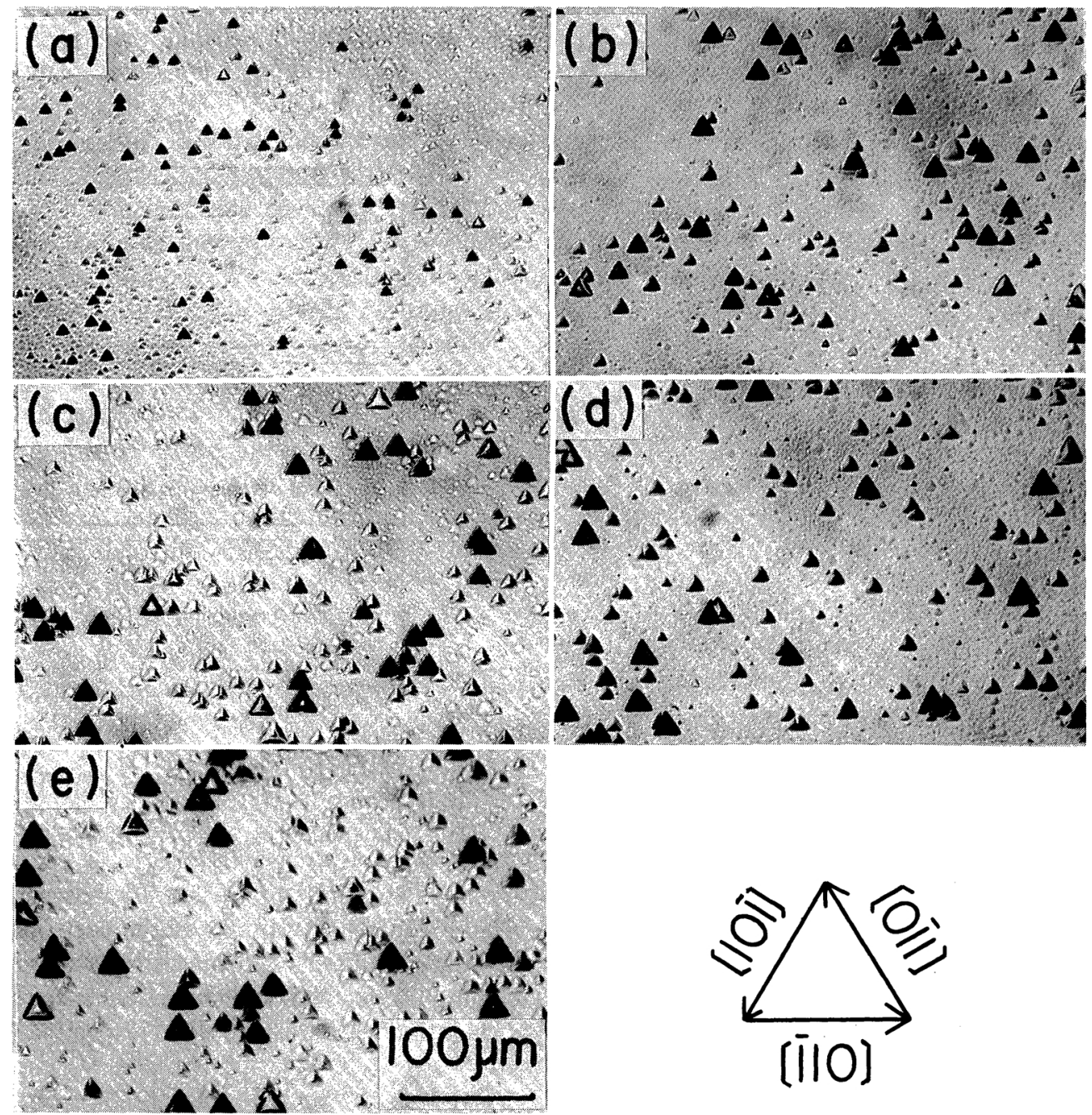

Fig. 3 Optical micrographs of dislocation etch pits formed on the (111) surface of as-grown $\mathrm{Cu}$ crystal after etching in Young's solution(11) at $290 \mathrm{~K}$ for (a) $5 \mathrm{~s}$, (b) $10 \mathrm{~s}$, (c) $15 \mathrm{~s}$, (d) $20 \mathrm{~s}$ and (e) $25 \mathrm{~s}$.

any etching time.

Dislocation etch pits were formed on the exposed (111) surface. Figure 3 shows optical micrographs of the dislocation etch pits at successive etching times. We find that triangular pits, of which contrasts are light, dark and mixed one, are revealed and that they become larger with increasing etching time. Previously, it has been shown that the dark and light pits may correspond to edge dislocations with positive and negative signs ${ }^{(9)}$. In the present work, three-dimensional shape and size of dark pits were examined by a replica electron micros- copy. Typical electron micrographs are shown in Fig. 4, from which it is seen that the etch pit forms a triangular pyramid having steps in its side surfaces and grows large without a shape change with an increase of the etching time. As illustrated schematically in Fig. 2, a threedimensional size of the etch pit can coveniently be measured by the depth $d$ and width $h$ which is taken as a length from a center of base triangle to its side centers. On electron micrographs magnified 1500-2000 times, the width $h$ was measured directly and the depth $d$ was calculated from the length of shadow against the 


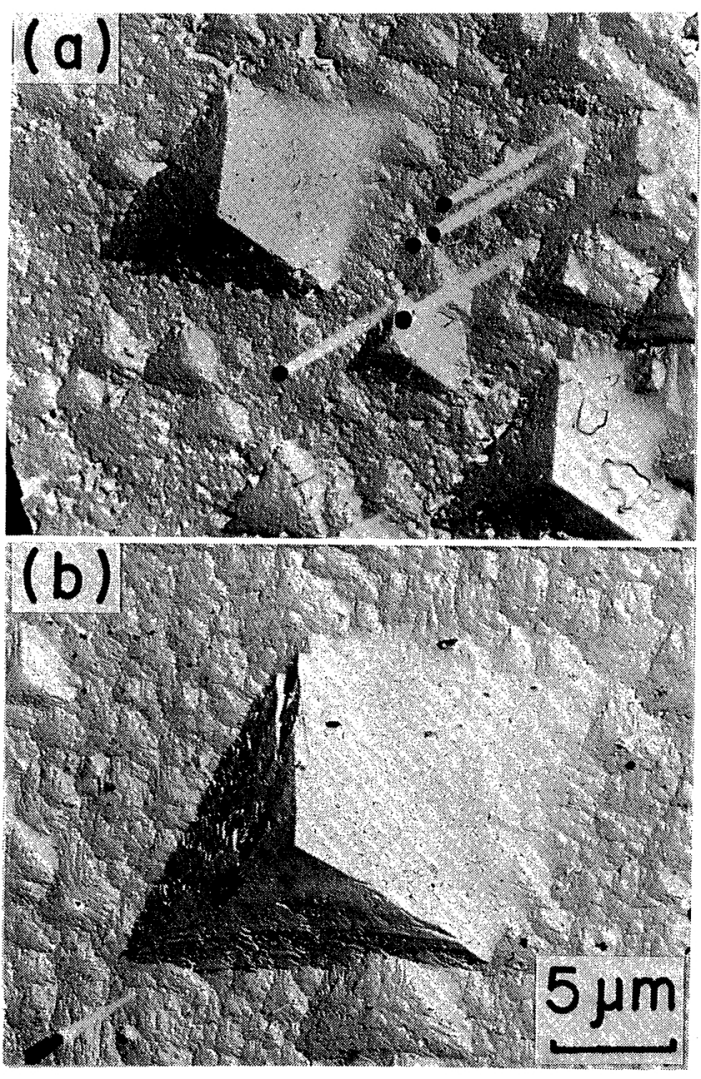

Fig. 4 Typical electron micrographs of dark etch pits formed on the (111) surface of as-grown $\mathrm{Cu}$ crystal after etching in Young's solution ${ }^{(11)}$ at $290 \mathrm{~K}$ for (a) $5 \mathrm{~s}$ and (b) $25 \mathrm{~s}$.

pit with the aid of that against latex spheres of known dimension. These measurements were made with 30 dark pits at every etching experiment, in all 90 pits for three etching experiments at the specified etching time. Using the measured values of $s, h$ and $d$, a net dissolved amount along a dislocation line, $D$, and that vertical to it, $H$, (see Fig. 2) were obtained, respectively, from

$$
D=s+d
$$

and

$$
H=(s+d) h / d
$$

\section{Experimental Results and Discussion}

Figure 5(a)-(c) shows the dissolved thickness of matrix surface $s$, the width $h$ and the depth $d$ of dark pits on the $\mathrm{Cu}$ (111) surface etched in
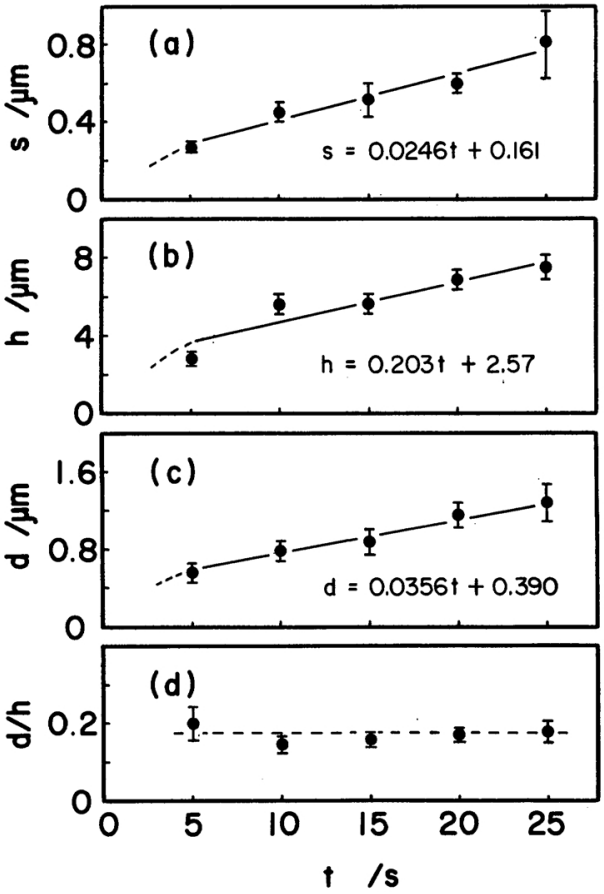

Fig. 5 Changes in (a) thickness of matrix surface dissolved $s$, (b) width $h$, (c) depth $d$ and (d) slope $d / h$ of dark etch pits with etching time $t$.

Young's solution for 5-25 s. Each value of $s$, $h$ and $d$ increases rapidly at the early stage of etching and after $5 \mathrm{~s}$ increases linearly with the etching time $t$. The time dependences for steady dissolution are expressed, respectively, by

and

$$
\left.\begin{array}{l}
s=\dot{s} t+s_{0}, \\
h=h t+h_{0} \\
d=\dot{d} t+d_{0},
\end{array}\right\}
$$

and the side slope $d / h$ of the etch pits can be written as ${ }^{(12)}$

$$
d / h=\frac{\dot{d} t+d_{0}}{h t+h_{0}}=\frac{\dot{d}}{\dot{h}}\left(1-\frac{t_{h}-t_{d}}{t+t_{h}}\right),
$$

where

$$
t_{h}=h_{0} / \dot{h} \text { and } t_{d}=d_{0} / \dot{d} .
$$

For $\left(t_{h}-t_{d}\right) /\left(t+t_{h}\right) \approx 0, d / h$ can be reduced to

$$
d / h \approx \dot{d} / h \text {. }
$$

By putting into eqs. (4) and (5) the experimental values $h=0.203 \mu \mathrm{m} \cdot \mathrm{s}^{-1}, \quad h_{0}=2.57 \mu \mathrm{m}, \quad \dot{d}=$ $0.0356 \mu \mathrm{m} \cdot \mathrm{s}^{-1}$ and $d_{0}=0.390 \mu \mathrm{m}$ as determined from Fig. 5 by the least square method, we obtain $\left(t_{h}-t_{d}\right) /\left(t+t_{h}\right)=0.1-0.05$ for $t=5-25 \mathrm{~s}$, 
indicating that eq. (6) is practically satisfied in the present case. This point is illustrated also in Fig. 5(a), in which the measured values of $d / h$ at each etching time coincide well with $\dot{d} / \hat{h}=$ 0.175 represented by the dashed line within $10 \%$. This means that the dislocation etch pit grows at a constant rate of $h$ and $\dot{d}$, keeping a similar shape as shown in Fig. 4. Previously, one of the authors (Watanabé) and his coworker $^{(12)}$ have studied the morphology of dislocation etch pits on the $\mathrm{Cu}$ (111) surface under potentiostatic etching in a solution containing $\mathrm{NaCl}, \mathrm{NaBr}$ and $\mathrm{CuCl}$ and found that $h$ and $d$ increased linearly with increasing $t$ while $d / h$ decreased with $t$ asymptotically to $\dot{d} / h$. Ives and Hirth ${ }^{(5)}$ measured the profile of dislocation etch pits on the LiF (100) surface etched in an aqueous solution containing $\mathrm{FeF}_{3}$, and found that $h$ increased linearly with $t$ but $d / h$ became large at the central portion of etch pit and took the maximum value after a prolonged etching. Regarding the time dependence of $h$ and $d$, an agreement exists between the present work and the previous ones, though regarding the time dependence of $d / h$, a disagreement arises between them. It seems probable that the disagreement might be caused by a difference in etching conditions. It is noted also that the dislocation etch pits presented here were surrounded by side surfaces which made a small angle of $0.175 \mathrm{rad}\left(10^{\circ}\right)$ with the (111) plane (see Fig. 5(d)), corresponding to a highindex crystallographic plane, in contrast to those of etch pits for orientation works, corresponding to the low-index crystallographic plane.

Now, the dissolution rate at dislocations on the $\mathrm{Cu}$ (111) surface etched in Young's solution will be estimated. The dissolution rate of the matrix surface, $v_{s}$, is estimated from Fig. 5(a) and given as $v_{s}=\dot{s}=0.025 \mu \mathrm{m} \cdot \mathrm{s}^{-1}$. Then, using the values of $\dot{s}, h$ and $\dot{d}$, the lateral dissolution rate normal to dislocation line, $v_{h}=$ $\mathrm{d} H / \mathrm{d} t$, and the vertical dissolution rate along dislocation line, $v_{d}=\mathrm{d} D / \mathrm{d} t$, are obtained, respectively, from

and

$$
\left.\begin{array}{l}
v_{h}=(\dot{s}+\dot{d}) h / d \\
v_{d}=(\dot{s}+\dot{d}),
\end{array}\right\}
$$
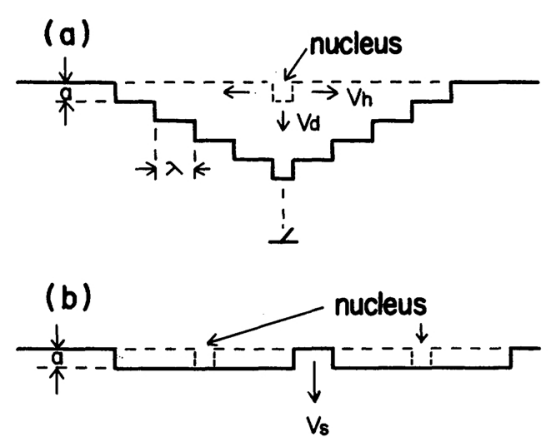

Fig. 6 Schematic illustration of dissolution by nucleation and growth of two-dimensional nuclei at (a) a dislocation site and (b) a matrix surface.

and are given as $v_{h}=0.34 \mu \mathrm{m} \cdot \mathrm{s}^{-1}$ and $v_{d}=$ $0.060 \mu \mathrm{m} \cdot \mathrm{s}^{-1}$. These values of $v_{h}$ and $v_{d}$ are 1.7 times as large as those of $v_{h} \approx h=0.203 \mu \mathrm{m}$. $\mathrm{s}^{-1}$ and $v_{d} \approx d=0.0356 \mu \mathrm{m} \cdot \mathrm{s}^{-1}$, which are derived in disregard of $\dot{s}$ in eq. (7). Accordingly, it is concluded that the dissolution of matrix surface must be taken into account to estimate the net dissolution rates at dislocation sites.

As stated in I, the formation of dislocation etch pits depends on the nucleation rate of twodimensional etch pit nuclei at a dislocation and the rate of motion of the resultant steps across the crystal surface. Let us denote, in Fig. 6(a), the height of a two-dimensional nucleus as $a$ (one atomic spacing). Then, the nucleation rate of the pit nucleus, $\dot{n}_{d}$, is given by

$$
\dot{n}_{d}=v_{d} / a \text {. }
$$

If we take $a=2.08 \times 10^{-4} \mu \mathrm{m}$ for $\mathrm{Cu}$ (111) spacing and the experimental value of $v_{d}=$ $0.060 \mu \mathrm{m} \cdot \mathrm{s}^{-1}$, we obtain $\dot{n}_{d}=2.9 \times 10^{2} \mathrm{~s}^{-1}$ from eq. (8), which indicates, in turn, that the etch-pit nucleus is formed at a time interval of $\tau_{d}=1 / \dot{n}_{d}=3.4 \times 10^{-3} \mathrm{~s}$ along the dislocation line. Then, a distance of $\langle 110\rangle$ step movement at a time interval $\tau_{d}$ is given by $\lambda=v_{h} \cdot \tau_{d}$, and hence $\lambda$ is estimated to be $1.2 \times 10^{-3} \mu \mathrm{m}$ by using the experimental value of $v_{h}=0.34 \mu \mathrm{m}$. $\mathrm{s}^{-1}$ and the above value of $\tau_{d}$.

Simultaneously with dissolution at a dislocation site, dissolution of dislocation-free matrix surface can occur by the formation of twodimensional nucleus with one atom height and the subsequent advance of the resultant steps across the surface, as shown in Fig. 6(b). Many 
nuclei are produced at random sites on the matrix surface, and they grow laterally by the advance of steps due to a removal of atoms from the kink sites, until the resultant neighboring holes are annihilated by collision and consequently one atomic layer is removed away from the matrix surface. Successive occurrence of these processes may lead to a layer by layer dissolution of the matrix surface. Schaarwächter ${ }^{(3)}$ deduced a kinetic equation for the above process, assuming that a two-dimensional nucleus would grow laterally forming a circular shape. However, some modification of his treatment is needed, in order that it may be applied to the present case, because a twodimensional nucleus formed on the $\mathrm{Cu}$ (111) surface is thought to grow laterally with $\langle 110\rangle$ steps forming a triangular shape similarly to the case of dissolution at a dislocation site.

Now, we assume that a two-dimensional nucleus is formed homogeneously at a rate of $\dot{n}$ on a unit area of matrix surface in a unit time. Then, the number of nuclei formed on the whole area of matrix surface $F_{0}$ during a period between $t^{\prime}$ and $t^{\prime}+\mathrm{d} t^{\prime}$ is given by $F_{0} \dot{n} \mathrm{~d} t^{\prime}$. Since each nucleus grows laterally with a rate of $v_{h}$ forming a triangular shape, the total area occupied by the resultant holes at a time $t\left(t>t^{\prime}\right)$, that is, the dissolved matrix surface area $F(t)$, is written as

$$
F(t)=\int_{t^{\prime}=0}^{t^{\prime}=t} 3 \sqrt{3} v_{h}^{2}\left(t-t^{\prime}\right)^{2} F_{0} \dot{n} \mathrm{~d} t .
$$

By equating $F(t)$ with $F_{0}$, the time required to dissolve the whole area of matrix surface, $\tau_{s}$, is expressed by

$$
\tau_{s}=3^{-1 / 6} \dot{n}^{-1 / 3} v_{h}^{-2 / 3} .
$$

Since the matrix surface dissolves into an etchant by one atomic spacing $a$ at successive intervals of $\tau_{s}$, the dissolution rate normal to the matrix surface is given by

$$
v_{s}=a / \tau_{s}
$$

From eqs. (10) and (11), we obtain

$$
\dot{n}=3^{-1 / 2} a^{-3} v_{s}^{3} v_{h}^{-2} \text {. }
$$

Taking $a=2.08 \times 10^{-4} \mu \mathrm{m}, v_{s}=0.025 \mu \mathrm{m} \cdot \mathrm{s}^{-1}$ and $v_{h}=0.34 \mu \mathrm{m} \cdot \mathrm{s}^{-1}$, we get $\dot{n}=8.7 \times$ $10^{6} \mu \mathrm{m}^{-2} \cdot \mathrm{s}^{-1}$ for a two-dimensional nucleation rate on a matrix surface and $\tau_{s}=8.4 \times$
$10^{-3} \mathrm{~s}$ for a time required to dissolve one atomic layer from a matrix surface. It is to be noted that $\tau_{s}$ may correspond to an average life time of steps during which they move laterally starting from nucleus before annihilation.

In summary, the distinction between the nucleation rate of two-dimensional etch-pit nucleus at a dislocation site and that at a dislocation-free site on $\mathrm{Cu}$ (111) surface has been found from a semi-quantitative analysis of the experimental data. However, it has been predicted from the nucleation theory for crystal dissolution that the formation energy of the critical two-dimensional nucleus at a dislocation site must be smaller than that at a matrix surface and that the size of the critical nucleus must be also so. In order to test these points, a temperature dependence of the two-dimensional nucleation rates must be examined. Hence, further experiments on this subject are in progress.

\section{Conclusion}

The (111) surface of $\mathrm{Cu}$ crystal was etched with Young's solution $\left(1 \mathrm{kmol} \cdot \mathrm{m}^{-3}\left(\mathrm{NH}_{4}\right)_{2} \mathrm{~S}_{2}\right.$ $\mathrm{O}_{8}, 6 \mathrm{kmol} \cdot \mathrm{m}^{-3} \mathrm{NH}_{4} \mathrm{OH}, 0.3 \mathrm{kmol} \cdot \mathrm{m}^{-3} \mathrm{NH}_{4}$ $\mathrm{Br})$ at $290 \mathrm{~K}$ for $5-25 \mathrm{~s}$, and the dissolution at dislocation sites and matrix surface was studied. The results are as follows.

(1) The width $h$ and depth $d$ of dislocation etch pits (dark pits) increased abruptly at an initial stage of etching and then increased at constant rate of $h$ and $\dot{d}$, respectively, keeping the side slope $d / h$ unchanged.

(2) The matrix surface dissolved also at a constant rate $\dot{s}$ after $5 \mathrm{~s}$ etching. The net dissolution rate along a dislocation line, $v_{d}$, and that vertical to the dislocation line, $v_{h}$, were estimated by taking account of the simultaneous dissolution of the matrix surface. The values of $v_{d}$ and $v_{h}$ obtained were found to be 1.7 times as large as the measured growth rates of the etch pits $\dot{d}$ and $h$, respectively, indicating that the dissolution of matrix surface was far from negligible in describing the dissolution at dislocation sites.

(3) Using the values of $v_{h}, v_{d}$ and $\dot{s}$, the nucleation rate of two-dimensional etch-pit nucleus at a dislocation site and that at a matrix surface were estimated to be $2.9 \times$ 
$10^{2} \mathrm{~s}^{-1}$ per dislocation and $8.7 \times 10^{6} \mu \mathrm{m}^{-2}$. $\mathrm{s}^{-1}$, respectively.

\section{Acknowledgment}

The authors wish to thank Messrs. K. Kikuchi and T. Sugawara for their assistance to the experiments.

\section{REFERENCES}

(1) J. J. Gilman, W. G. Johnston and G. W. Sears: J. Appl. Phys., 29 (1958), 747.

(2) N. Cabrera and M. M. Levin: Phil. Mag., 1 (1956), 450.

(3) W. Schaarwächter: Phys. Status Solidi, 12 (1965), 375,865 .

(4) R. B. Heiman: Auflösung von Kristallen, Springer-
Verlag, Wien, (1975), p. 1.

(5) M. B. Ives and J. P. Hirth: J. Chem. Phys., 33 (1960), 517.

(6) L. Jasper and W. Schaarwächter: Z. Metallk., 57 (1966), 661.

(7) V. N. Rozahanskii, E. V. Parvova, V. N. Stepanova and A. A. Predvoditelev: Soviet Phys. Crystallog., 6 (1962), 564.

(8) N. A. Toropov and Yu. P. Udalov: Soviet Phys. Dokl., 10 (1965), 259.

(9) J. Watanabé and S. Sugawara: Trans. JIM, 19 (1978), 511.

(10) Y. Imashimizu and J. Watanabé: Trans. JIM, 24 (1983), 1.

(11) F. W. Young, Jr.: J. Appl. Phys., 32 (1961), 192.

(12) Y. Imashimizu and J. Watanabé: Trans. JIM, 24 (1983), 791. 